\title{
Non-Equilibrium Thermodynamics of Ferroelectric Phase Transitions
}

\author{
Shu-Tao Ai \\ Linyi Normal University \\ People's Republic of China
}

\section{Introduction}

It is well known that the Landau theory of continuous phase transitions is a milestone in the process of the development of phase transition theories. Though it does not tally with the nature of phase transitions in the critical regions, the Landau theory as a phenomenological one has been very successful in many kinds of phase transitions such as the ferroelectric phase transitions, i.e. the vast studies centering on it have been carried out. In the ferroelectric case, we should pay attention to the Landau theory extended by A.F. Devonshire to the first-order phase transitions (Devonshire, 1949; Devonshire, 1951; Devonshire, 1954). This daring act was said to be successful.

However, the Landau theory is based on the equilibrium (reversible) thermodynamics in essence. Can it deal with the outstanding irreversible phenomenon of first-order ferroelectric phase transitions, which is the "thermal hysteresis"? The Landau-Devonshire theory attributes the phenomenon to a series of metastable states existing around the Curie temperature $T_{C}$. In principle, the metastable states are not the equilibrium ones and can not be processed by using the equilibrium thermodynamics. Therefore, we believe that the extension of Devonshire is problematic though it is successful in mathematics. The real processes of phase transition were distorted. In Section 2, we will show the unpleasant consequence caused by the metastable states hypothesis, and the evidence for the non-existence of metastable states, i.e. the logical conflict. Then in Section 3, we will show the evidence (experimental and theoretical) for the existence of stationary states to a ferroelectric phase transition. In Section 4 and 5, we will give the non-equilibrium (irreversible) thermodynamic description of phase transitions, which eliminates the unpleasant consequence caused by the metastable states hypothesis. At last, in Section 6 we will give the non-equilibrium thermodynamic explanation of the irreversibility of ferroelectric phase transitions, i.e. the thermal hysteresis and the domain occurrences in ferroelectrics.

\section{Limitations of Landau-Devonshire theory}

The most outstanding merit of Landau-Devonshire theory is that the Curie temperature and the spontaneous polarization at Curie temperature can be determined simply. However, in the Landau-Devonshire theory, the path of a first-order ferroelectric phase transition is believed to consist of a series of metastable states existing around the Curie temperature $T_{C}$. This is too difficult to believe because of the difficulties encountered (just see the following). 


\subsection{Unpleasant consequence caused by metastable states hypothesis}

Basing on the Landau-Devonshire theory, we make the following inference. Because of the thermal hysteresis, a first-order ferroelectric phase transition must occur at another temperature, which is different from the Curie temperature. The state corresponding to the mentioned temperature (actual phase transition temperature) is a metastable one. Since the unified temperature and spontaneous polarization can be said about the metastable state, we neglect the heterogeneity of system actually. In other words, every part of the system, i.e. either the surface or the inner part, is of equal value physically. When the phase transition occurs at the certain temperature, every part of the system absorbs or releases the latent heat simultaneously by a kind of action at a distance. (The concept arose in the electromagnetism first. Here, it maybe a kind of heat transfer). Otherwise, the heat transfer in system, with a finite rate, must destroy the homogeneity of system and lead to a non-equilibrium thermodynamic approach. The unpleasant consequence, i.e. the action at a distance should be eliminated and the life-force should be bestowed on the non-equilibrium thermodynamic approach.

In fact, a first-order phase transition process is always accompanying the fundamental characteristics, called the co-existence of phases and the moving interface (phase boundary). This fact reveals that the phase transition at various sites can not occur at the same time. Yet, the phase transition is induced by the external actions (the absorption or release of latent heat). It conflicts sharply with the action at a distance.

\subsection{Evidence for non-existence of metastable states: logical conflict}

In the Landau-Devonshire theory, if we neglect the influence of stress, the elastic Gibbs energy $G_{1}$ can be expressed with a binary function of variables, namely the temperature $T$ and the electric displacement $D$ (As $G_{1}$ is independent of the orientation of $\boldsymbol{D}$, here we are interested in the magnitude of $\boldsymbol{D}$ only)

$$
G_{1}=g_{1}(T, D)
$$

The long-standing, close correlation between the analytical dynamics and the thermodynamics implies that Equation (1) can be taken as a scleronomic constraint equation

$$
f_{1}\left(G_{1}, T, D\right)=G_{1}-g_{1}(T, D)=0
$$

where $G_{1}, T, D$ are the generalized displacements. The possible displacements $d G_{1}, d T$ and $\mathrm{d} D$ satisfy the following equation

$$
\mathrm{d} G_{1}-\frac{\partial g_{1}}{\partial T} \mathrm{~d} T-\frac{\partial g_{1}}{\partial D} \mathrm{~d} D=0
$$

In the Landau-Devonshire theory, the scleronomic constraint equation, i.e. Equation (1) is expressed in the form of the power series of $D$ (For simplicity, only the powers whose orders are not more than six are considered)

$$
G_{1}=G_{10}+\frac{1}{2} \alpha D^{2}+\frac{1}{4} \beta D^{4}+\frac{1}{6} \gamma D^{6}
$$

where $\alpha, \beta, \gamma$ are the functions of $T$, and $G_{10}$ is the elastic Gibbs energy of paraelectric phase. The relation between $G_{1}$ and $D$ at various temperatures, which belongs to first-order 
phase transition ferroelectrics is represented graphically in Figure 1. The electric displacements which correspond with the bilateral minima of $G_{1}$ are identified as $\pm D^{*}$, and the electric displacement which corresponds with the middle minimum of $G_{1}$ equals zero. The possible electric displacements should be the above ones which correspond with all the minima of $G_{1}$.

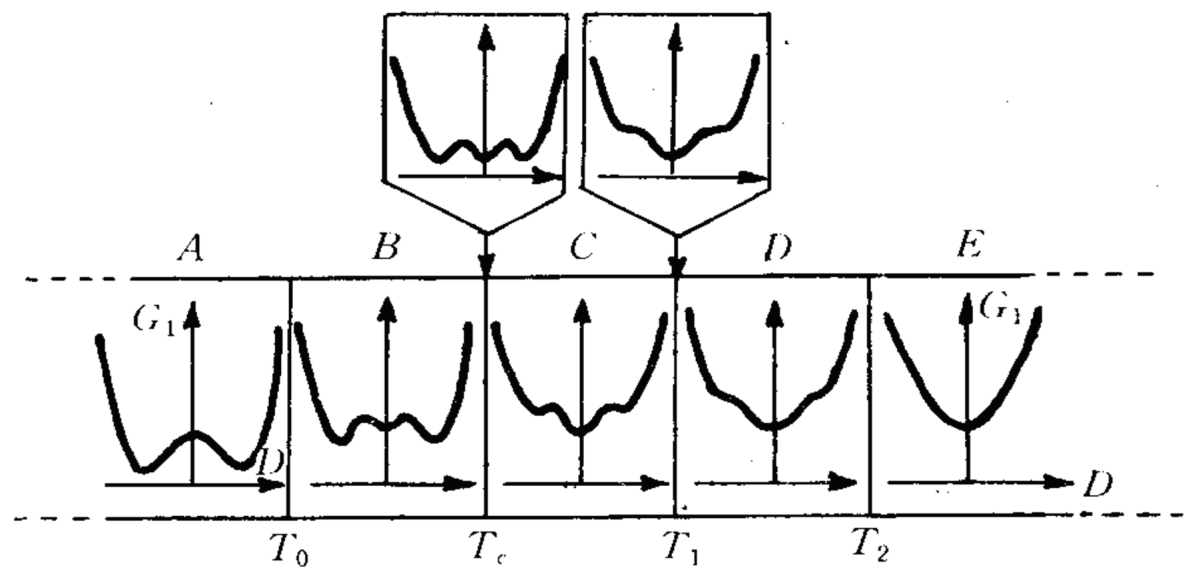

Fig. 1. The relation between elastic Gibbs energy $G_{1}$ and zero field electric displacement $D$ belongs to the ferroelectrics at various temperatures, which undergoes a first-order phase transition (Zhong, 1996).

Equivalently, imposed on the generalized displacements $G_{1}, T, D$ is a constraint, which is

$$
\frac{\partial G_{1}}{\partial D}=0
$$

So, the possible displacement $\mathrm{d} D$ should be the following:

$$
\mathrm{d} D^{*},-\mathrm{d} D^{*}, 0-\left( \pm D^{*}\right)=\mp D^{*}, \pm D^{*}-0= \pm D^{*}, 0 \text {. }
$$

After all, if our discussions are limited in the equilibrium thermodynamics strictly, there must be the third constraint, i.e. the equilibrium $D$ and $T$ should satisfy

$$
h(D, T)=0
$$

where $h$ is a binary function of the variables $D$ and T. It can be determined by the principle of minimum energy

$$
G_{1}=\min
$$

for certain $T$. Then, the metastable states are excluded. Thus, the thermal hysteresis does not come into being. The corollary conflicts with the fact sharply. This reveals that the first-order ferroelectric phase transition processes must not be reversible at all so as not to be dealt with by using the equilibrium thermodynamics. 
How can this difficulty be overcome? An expedient measure adopted by Devonshire is that the metastable states are considered. However, do they really exist?

Because the metastable states are not the equilibrium ones, the relevant thermodynamic variables or functions should be dependent on the time $t$. In addition, the metastable states are close to equilibrium, so the heterogeneity of system can be neglected. Here, the elastic Gibbs energy $G_{1}^{\prime}$ should be

$$
G_{1}^{\prime}=g_{2}(T, D, t)
$$

For the same reason as was mentioned above, Equation (8) can be regarded as a rheonomic constraint on the generalized displacements $G_{1}^{\prime}, T, D$

$$
f_{2}\left(G_{1}^{\prime}, T, D, t\right)=G_{1}^{\prime}-g_{2}(T, D, t)=0
$$

In this case, the possible displacements $\mathrm{d} G_{1}^{\prime}, \mathrm{d} T$ and $\mathrm{d} D$ satisfy the following equation

$$
\mathrm{d} G_{1}^{\prime}-\frac{\partial g_{2}}{\partial T} \mathrm{~d} T-\frac{\partial g_{2}}{\partial D} \mathrm{~d} D-\frac{\partial g_{2}}{\partial t} \mathrm{~d} t=0
$$

Comparing Equation (3) with Equation (10), we may find that the possible displacements here are not the same as those in the former case which characterize the metastable states for they satisfy the different constraint equations, respectively. (In the latter case, the possible displacements are time-dependent, whereas in the former case they are not.). Yet, the integral of possible displacement $\mathrm{d} D$ is the possible electric displacement in every case. The possible electric displacements which characterize one certain metastable state vary with the cases. A self-contradiction arises. So the metastable states can not come into reality.

What are the real states among a phase transition process? In fact, both the evolution with time and the spatial heterogeneity need to be considered when the system is out of equilibrium (Gordon, 2001; Gordon et al., 2002; Ai, 2006; Ai, 2007). Just as what will be shown in Section 3, the real states should be the stationary ones, which do not vary with the time but may be not metastable.

\section{Real path: existence of stationary states}

The real path of a first-order ferroelectric phase transition is believed by us to consist of a series of stationary states. At first, this was conjectured according to the experimental results, then was demonstrated reliable with the aid of non-equilibrium variational principles.

\subsection{Conjecture of stationary states based on experiments}

Because in the experiments the ferroelectric phase transitions are often achieved by the quasi-static heating or cooling, we conjectured that they are stationary states processes (Ai, 2006). The results on the motion of interface in ferroelectrics and antiferroelectrics support our opinion (Yufatova et al., 1980; Dec, 1988; Dec \& Yurkevich, 1990). From Figure 2, we may find that the motion of interface is jerky especially when the average velocity $v_{a}$ is small. A sequence of segments of time corresponding to the states of rest may be found. This reveals that in these segments of time (characteristic time of phase transition) the stationary 


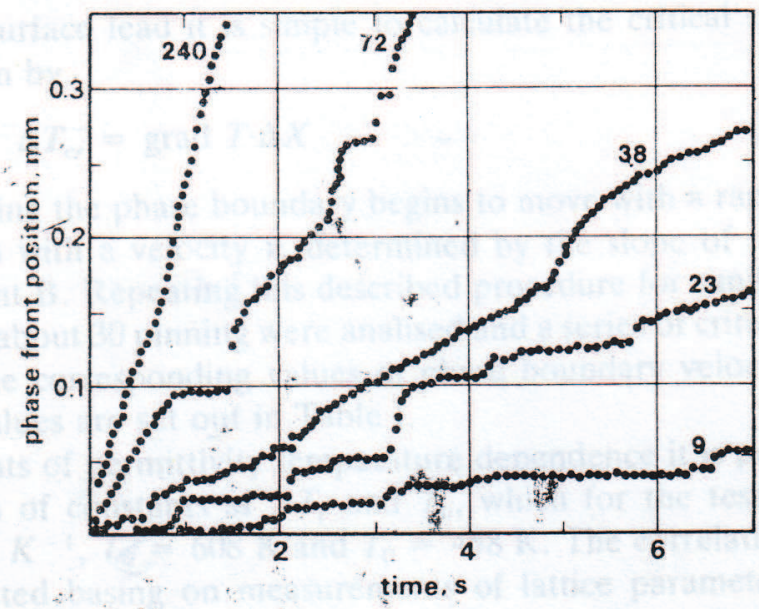

Fig. 2. The position of phase boundary as a function of time for $\mathrm{NaNbO}_{3}$ single crystal for the various values of average velocity $v_{a}$ (the values in $\mu \mathrm{m} / \mathrm{s}$ given against the curves). (Dec \& Yurkevich, 1990).

distributions of temperature, heat flux, stress, etc. may be established. Otherwise, if the motion of interface is continuous and smooth, with the unceasing moving of interface (where the temperature is $T_{C}$ ) to the inner part, the local temperature of outer part must change to keep the temperature gradient $\nabla T$ of this region unchanged for it is determined by $\pm l \rho \boldsymbol{v}^{\prime}=\boldsymbol{J}_{q}^{\text {diff }}=-\kappa \nabla T$, where $l$ is the latent heat (per unit mass), $\rho$ is the mass density, $\boldsymbol{v}^{\prime}$ is the velocity of interface (where the phase transition is occurring), $\boldsymbol{J}_{\boldsymbol{q}}^{\text {diff }}$ is the diffusion of heat, i.e. heat conduction, $\kappa$ is the thermal conductivity (and maybe a tensor). Then, the states are not stationary.

\subsection{Theoretical evidence based on non-equilibrium variational principles}

The non-equilibrium variational principles are just the analogue and generalization of the variational principles in the analytical dynamics. The principle of least dissipation of energy, the Gauss's principle of least constraint and the Hamiltonian prinple etc. in the nonequilibrium thermodynamics play the fundamental roles as those in the analytical dynamics. They describe the characteristics of stationary states or determine the real path of non-equilibrium processes.

For the basic characteristic of non-equilibrium processes is the dissipation of energy, we define the dissipation function $\phi$ as

$$
\phi=\sigma_{s}-\pi
$$

where $\sigma_{s}$ is the rate of local entropy production and $\pi$ is the external power supply (per unit volume and temperature). Let $\lambda=\left\{\lambda_{i}\right\}$ represents the set of extensive, pseudothermodynamic variables and its derivative with the time $t$, i.e. $\dot{\lambda}$ represents the thermodynamic fluxes. And we define a thermodynamic force $X_{T i}$ and a dissipative force $X_{D i}$ as 


$$
\begin{aligned}
& X_{T i}(\lambda)=\frac{\partial \sigma_{s}}{\partial \dot{\lambda}_{i}} \\
& X_{D i}(\dot{\lambda})=\frac{\partial \phi}{\partial \dot{\lambda}_{i}}
\end{aligned}
$$

Let $\xi=\left\{\xi_{i}\right\}$ represent the deviation from a given non-equilibrium stationary state and provided it is small

$$
\begin{gathered}
\xi=\lambda-\lambda^{(0)} \\
|\xi| \lambda \mid<<1
\end{gathered}
$$

where $\lambda^{(0)}$ corresponds with the stationary state. The thermodynamic force can be expanded in terms of the deviation

$$
X_{T i}(\lambda)=\chi_{T i}^{(0)}+\chi_{T i}^{(1)}(\xi)+\chi_{T i}^{(2)}\left(\xi^{2}\right)+\cdots
$$

The linear variation in the thermodynamic force can be written as

$$
\chi_{T i}^{(1)}(\xi)=S_{i j} \xi_{j}
$$

(sum up with respect to $j$ ). In certain diagonal representation, Equation (17a) can be rewritten as

$$
\chi_{T i}^{(1)}(\xi)=S_{i} \xi_{i}
$$

(not sum up with respect to $i$ ). $S$ is a negatively-definite matrix.

The dissipation function $\phi$ can be expanded in terms of the deviation

$$
\phi=\phi^{(0)}+\delta \phi+\delta^{2} \phi+\cdots
$$

Because of the least dissipation of energy at stationary states, the linear variation in dissipation function should be zero

$$
\delta \phi=0
$$

The quadratically non-linear variation in dissipation function can be written as

$$
\delta^{2} \phi=\frac{1}{2} R_{i j} \dot{\xi}_{i} \dot{\xi}_{j}
$$

(sum up with respect to $i, j$ ). For the same reason, it must be non-negative. So, $R$ is a positively-definite matrix. According to Equation (13), the dissipation force adopts the following form

$$
X_{D i}=\chi_{D i}^{(1)}(\dot{\xi})+\cdots
$$


with

$$
\chi_{D i}^{(1)}(\dot{\xi})=R_{i j} \dot{\xi}_{j}
$$

(sum up with respect to $j$ ). In the certain diagonal representation, Equation (22a) can be rewritten as

$$
\chi_{D i}^{(1)}(\dot{\xi})=R_{i} \dot{\xi}_{i}
$$

(not sum up with respect to $i$ ).

In view of Equation (14) and (16), the entropy production $\sigma_{s}$ is given by the expansion

Correspondingly

$$
\begin{aligned}
& \sigma_{s}=\sigma_{s}^{(0)}+\delta \sigma_{s}+\delta^{2} \sigma_{s}+\cdots \\
& =X_{T i} \cdot \dot{\lambda}_{i} \\
& =\left(\chi_{T i}^{(0)}+\chi_{T i}^{(1)}+\chi_{T i}^{(2)}+\cdots\right) \cdot\left(\dot{\lambda}_{i}^{(0)}+\dot{\xi}_{i}\right) \\
& =\chi_{T i}^{(0)} \cdot \dot{\lambda}_{i}^{(0)}+\chi_{T i}^{(0)} \cdot \dot{\xi}_{i}+\chi_{T i}^{(1)} \cdot \dot{\lambda}_{i}^{(0)} \\
& +\chi_{T i}^{(1)} \cdot \dot{\xi}_{i}+\chi_{T i}^{(2)} \cdot \dot{\lambda}_{i}^{(0)}+\cdots
\end{aligned}
$$

$$
\begin{gathered}
\sigma_{s}^{(0)}=\chi_{T i}^{(0)} \cdot \dot{\lambda}_{i}^{(0)} \\
\delta \sigma_{s}=\chi_{T i}^{(0)} \cdot \dot{\xi}_{i}+\chi_{T i}^{(1)} \cdot \dot{\lambda}_{i}^{(0)} \\
\delta^{2} \sigma_{s}=\chi_{T i}^{(1)} \cdot \dot{\xi}_{i}+\chi_{T i}^{(2)} \cdot \dot{\lambda}_{i}^{(0)}
\end{gathered}
$$

(sum up with respect to $i$ ). Due to the minimum entropy production at stationary states, the linear variation in the entropy production should be zero

$$
\delta \sigma_{s}=0
$$

Similarly, the power function $\pi$ can be expanded about the stationary state, i.e.

$$
\pi=\pi^{(0)}+\delta \pi+\delta^{2} \pi+\cdots
$$

And correspondingly, we can define

$$
X_{E i}(\lambda)=\frac{\partial \pi}{\partial \dot{\lambda}_{i}}=\chi_{E i}^{(0)}+\chi_{E i}^{(1)}+\chi_{E i}^{(2)}+\cdots
$$

Thus, repeating the steps between Equations (23) and (26), we have

$$
\begin{gathered}
\pi^{(0)}=\chi_{E i}^{(0)} \cdot \dot{\lambda}_{i}^{(0)} \\
\delta \pi=\chi_{E i}^{(0)} \cdot \dot{\xi}_{i}+\chi_{E i}^{(1)} \cdot \dot{\lambda}_{i}^{(0)}
\end{gathered}
$$




$$
\delta^{2} \pi=\chi_{E i}^{(1)} \cdot \dot{\xi}_{i}+\chi_{E i}^{(2)} \cdot \dot{\lambda}_{i}^{(0)}
$$

(sum up with respect to $i$ ).

Introducing the expansions, i.e. Equations (18), (23) and (28) into Equation (11) and setting equal terms of the same order, we obtain at first order

$$
\delta \pi=\chi_{E i}^{(0)} \cdot \dot{\xi}_{i}+\chi_{E i}^{(1)} \cdot \dot{\lambda}_{i}^{(0)}=0
$$

(sum up with respect to $i$ ). Further, we may find that

$$
\frac{\chi_{T i}^{(0)}}{\chi_{T i}^{(1)}}=\frac{\chi_{E i}^{(0)}}{\chi_{E i}^{(1)}}=-\frac{\dot{\lambda}_{i}^{(0)}}{\dot{\xi}_{i}}
$$

At second order we find

$$
\frac{1}{2} \chi_{D i}^{(1)}=\chi_{T i}^{(1)}-\chi_{E i}^{(1)}
$$

(in Equations (34), (35), not sum up with respect to $i$ ).

By analogy, here we can give the thermodynamic Gauss's principle of least constraint which makes the system choose a real path. Let $X_{T}$ be the active force and $-X_{E}$ be the constraining force. The thermal inertia may be $R_{i}$. Then the constraint $Z$ can be defined as

$$
Z=\frac{1}{2 R_{i}}\left(X_{D i}-X_{T i}\right)^{2}=\frac{1}{2 R_{i}} X_{E i}^{2}
$$

(sum up with respect to $i$ ). The real path makes $Z$ be a minimum, i.e.

$$
\delta Z=0
$$

Assume that

$$
\chi_{E i}^{(1)}=V_{i j} \xi_{j}
$$

(sum up with respect to $j$ ) and in the diagonal representation

$$
\chi_{E i}^{(1)}=V_{i} \xi_{i}
$$

(not sum up with respect to $i$ ). Henceforth, we will make some discussions in two cases separately.

No external power supply. Then

$$
X_{E i}=0
$$

According to Equation (35), at second order level, we have

$$
\frac{1}{2} \chi_{D i}^{(1)}=\chi_{T i}^{(1)}
$$

In view of Equations (17b) and (22b), we obtain the evolution of deviation $\xi_{i}$ 


$$
\xi_{i}=\xi_{i}(0) e^{\frac{2 S_{i}}{R_{i}} t}
$$

where $\xi_{i}(0)$ is the initial value of $\xi_{i}$. Equation (41) defines the real path with the addition that $R_{i}$ should be a suitable value $R_{i}^{*}$. It can be determined by Equations (34) and (41)

$$
R_{i}^{*}=-\frac{2 \chi_{T i}^{(0)}}{\dot{\lambda}_{i}^{(0)}}
$$

The external power supply exists. Similarly, we can obtain the evolution of deviation $\xi_{i}$

$$
\xi_{i}=\xi_{i}(0) e^{\frac{2\left(S_{i}-V_{i}\right)}{R_{i}} t}
$$

If $V_{i}, R_{i}$ assume the suitable values $V_{i}^{* *}, R_{i}^{* *}$, the system choose a real path. They can be determined by Equations (34) and (43)

$$
\begin{gathered}
V_{i}^{* *}=\frac{\chi_{E i}^{(0)}}{\chi_{T i}^{(0)}} S_{i} \\
R_{i}^{* *}=\frac{2\left(\chi_{E i}^{(0)}-\chi_{T i}^{(0)}\right)}{\dot{\lambda}_{i}^{(0)}}
\end{gathered}
$$

(in every equation from Equation (39) to (45), not sum up with respect to $i$ ).

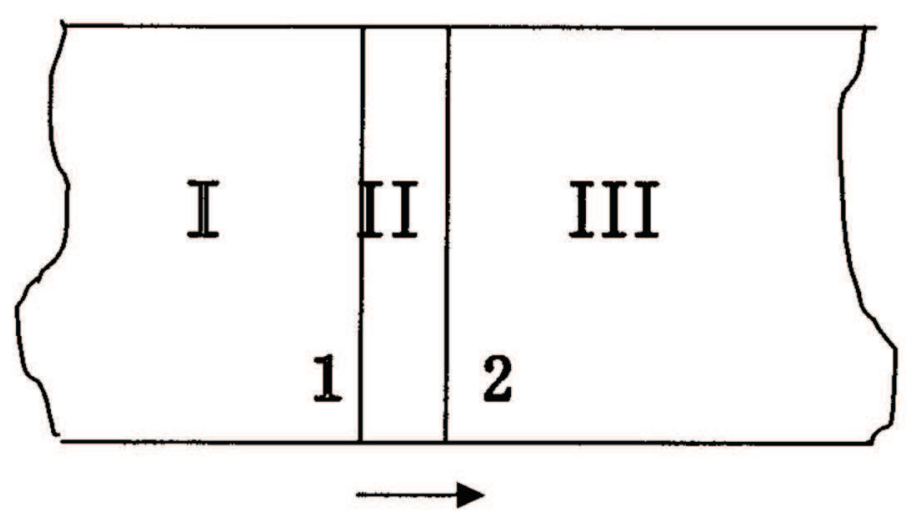

Fig. 3. Three types of regions and their interfaces in the ferroelectric-paraelectric system in which a first-order phase transition is occurring. (Ai et al., 2008)

Both the real paths in the two cases reveal that the deviations decrease exponentially when the system regresses to the stationary states. Stationary states are a kind of attractors to nonequilibrium states. The decreases are steep. So the regressions are quick. It should be noted that we are interested in calculating the change in the generalized displacements during a 
macroscopically small time interval. In other words, we are concerned with the determination of the path of an irreversible process which is described in terms of a finite difference equation. In the limit as the time interval is allowed to approach zero, we obtain the variational equation of thermodynamic path.

So, if the irreversible process is not quick enough, it can be regarded as the one that consists of a series of stationary states. The ferroelectric phase transitions are usually achieved by the quasi-static heating or cooling in the experiments. So, the processes are not quick enough to make the states deviate from the corresponding stationary states in all the time. In Figure 3, three types of regions and their interfaces are marked I, II, III, 1, 2 respectively. The region III where the phase transition will occur is in equilibrium and has no dissipation. In the region I where the phase transition has occurred, there is no external power supply, and in the region II (i.e. the paraelectric-ferroelectric interface as a region with finite thickness instead of a geometrical plane) where the phase transition is occurring, there exists the external supply, i.e. the latent heat (per unit volume and temperature). According to the former analysis in the two cases, we may conclude that they are in stationary states except for the very narrow intervals of time after the sudden lose of phase stability.

\section{Thermo-electric coupling}

In the paraelectric-ferroelectric interface dynamics induced by the latent heat transfer (Gordon, 2001; Gordon et al., 2002), the normal velocity of interface $v_{n}$ was obtained

$$
v_{n}=\frac{1}{l \rho}\left[k_{f e r}(\nabla T)_{f e r}-k_{p a r}(\nabla T)_{p a r}\right] \cdot \boldsymbol{n}
$$

where $l$ is the latent heat (per unit mass), $\rho$ is the density of metastable phase (paraelectric phase), $k_{f e r}$ is the thermal conductivity coefficient of ferroelectric phase, $k_{\text {par }}$ is the thermal conductivity coefficient of paraelectric phase, $(\nabla T)_{f e r}$ is the temperature gradient in ferroelectric phase part, $(\nabla T)_{\text {par }}$ is the temperature gradient in paraelectric phase part, $\boldsymbol{n}$ is the unit vector in normal direction. The temperature gradients can be studied from the point of view that a ferroelectric phase transition is a stationary, thermo-electric coupled transport process (Ai, 2006).

\subsection{Local entropy production}

In the thermo-electric coupling case, the Gibbs equation was given as the following

$$
\mathrm{T} \mathrm{d} s=\mathrm{d} u-\boldsymbol{E} \cdot \mathrm{d} \boldsymbol{D}-\sum_{i} \mu_{i} \mathrm{~d} n_{i}
$$

where $T, \boldsymbol{E}, \boldsymbol{D}$ is the temperature, the electric field intensity and the electric displacement within a random small volume, respectively; $s, u, \mu_{i}, n_{i}$ is the entropy density, the internal energy density, the chemical potential and the molar quantity density in the small volume, respectively. And there, it was assumed that the crystal system is mechanically-free (No force is exerted on it). Differentiating Equation (47) and using the following relations

$$
\frac{\partial u}{\partial t}+\nabla \cdot J_{u}=0
$$




$$
\begin{gathered}
\frac{\partial \boldsymbol{D}}{\partial t}=\frac{\partial\left(\varepsilon_{0} \boldsymbol{E}+\boldsymbol{P}\right)}{\partial t}=\boldsymbol{J}_{P} \\
\frac{\partial n_{i}}{\partial t}+\nabla \cdot \boldsymbol{J}_{n i}=0
\end{gathered}
$$

we have

$$
\frac{\partial s}{\partial t}=-\frac{1}{T} \nabla \cdot \boldsymbol{J}_{u}-\frac{\boldsymbol{E}}{T} \cdot \boldsymbol{J}_{P}+\sum_{i} \frac{\mu_{i}}{T} \nabla \cdot \boldsymbol{J}_{n i}
$$

where $\boldsymbol{J}_{u}, \boldsymbol{J}_{P}, \boldsymbol{J}_{n i}$ is the energy flux, the polarization current and the matter flux, respectively; $\boldsymbol{P}$ is the polarization. $\boldsymbol{J}_{u}$ should consist of three parts: the energy flux caused by the heat conduction, the energy flux caused by the charge transport, the energy flux caused by the matter transport

$$
\boldsymbol{J}_{u}=\boldsymbol{J}_{q}+\phi \boldsymbol{J}_{P}+\sum_{i} \mu_{i} \boldsymbol{J}_{n i}
$$

where $\boldsymbol{J}_{q}$ is the heat flux, $\phi$ is the electrical potential and satisfies

$$
\boldsymbol{E}=-\nabla \phi
$$

Then we deduce the following

$$
\frac{\partial s}{\partial t}=-\nabla \cdot\left(\frac{\boldsymbol{J}_{q}+\phi \boldsymbol{J}_{P}}{T}\right)+\boldsymbol{J}_{q} \cdot \nabla\left(\frac{1}{T}\right)+\boldsymbol{J}_{P} \cdot \nabla\left(\frac{\phi}{T}\right)-\sum_{i} \frac{\boldsymbol{J}_{n i}}{T} \cdot \nabla \mu_{i}
$$

If we define a entropy flux $\boldsymbol{J}_{s}$ and a rate of local entropy production $\sigma_{s}$ as

$$
\begin{gathered}
\boldsymbol{J}_{s}=\frac{\boldsymbol{J}_{q}+\phi \boldsymbol{J}_{P}}{T} \\
\sigma_{s}=\boldsymbol{J}_{q} \cdot \nabla\left(\frac{1}{T}\right)+\boldsymbol{J}_{P} \cdot \nabla\left(\frac{\phi}{T}\right)-\sum_{i} \frac{\boldsymbol{J}_{n i}}{T} \cdot \nabla \mu_{i}
\end{gathered}
$$

Equation (54) can be written as

$$
\frac{\partial s}{\partial t}+\nabla \cdot \boldsymbol{J}_{s}=\sigma_{s}
$$

This is the local entropy balance equation. We know, the system is in the crystalline states before and after a phase transition so that there is no diffusion of any kind of particles in the system. So, $\boldsymbol{J}_{n i}=0$. The local entropy production can be reduced as

$$
\sigma_{s}=\boldsymbol{J}_{q} \cdot \nabla\left(\frac{1}{T}\right)+\boldsymbol{J}_{P} \cdot \nabla\left(\frac{\phi}{T}\right)
$$

We know the existence of ferroics is due to the molecular field. It is an inner field. So we must take it into account. Here, the electric field should be the sum of the outer electric field $\boldsymbol{E}_{o}$ and the inner electric field $\boldsymbol{E}_{i}$ 


$$
\boldsymbol{E}=\boldsymbol{E}_{o}+\boldsymbol{E}_{i}
$$

Correspondingly, there are the outer electrical potential $\phi_{o}$ and the inner electrical potential $\phi_{i}$ and they satisfy

$$
\begin{gathered}
\boldsymbol{E}_{o}=-\nabla \phi_{o} \\
\boldsymbol{E}_{i}=-\nabla \phi_{i}
\end{gathered}
$$

If the outer electric field is not applied, $\phi_{0}$ can be a random constant. There is no harm in letting the constant equal to zero. Then the entropy production equals

$$
\sigma_{s}=\boldsymbol{J}_{q} \cdot \nabla\left(\frac{1}{T}\right)+\boldsymbol{J}_{P} \cdot \nabla\left(\frac{\phi_{i}}{T}\right)
$$

According to the crystal structures of ferroelectrics (Lines \& Glass, 1977), we know the polarization current $\boldsymbol{J}_{P}$ originates from the displacement or ordering of ions in ferroelectrics. We may consider it as the transport of charges influenced by the inner electric field.

\subsection{Description of phase transitions}

Assume the outer electric field is not applied. Here are the thermodynamic fluxes $\boldsymbol{J}_{q}, \boldsymbol{J}_{P}$ and the corresponding thermodynamic forces $\boldsymbol{X}_{q}, \boldsymbol{X}_{P}$

$$
\begin{aligned}
& \boldsymbol{X}_{q}=\nabla\left(\frac{1}{T}\right) \\
& \boldsymbol{X}_{P}=\nabla\left(\frac{\phi_{i}}{T}\right)
\end{aligned}
$$

$\boldsymbol{J}_{i}$ can be expanded linearly with $\boldsymbol{X}_{j}(i, j=q, P)$

$$
\begin{aligned}
& \boldsymbol{J}_{q}=\boldsymbol{L}_{q q} \cdot \boldsymbol{X}_{q}+\boldsymbol{L}_{q P} \cdot \boldsymbol{X}_{P} \\
& \boldsymbol{J}_{P}=\boldsymbol{L}_{P q} \cdot \boldsymbol{X}_{q}+\boldsymbol{L}_{P P} \cdot \boldsymbol{X}_{P}
\end{aligned}
$$

where $\boldsymbol{L}_{q q}, \boldsymbol{L}_{q P}, \boldsymbol{L}_{P q}$ and $\boldsymbol{L}_{P P}$ are the transport coefficients, which are four second-order tensors. The Onsager relations tell us (Lavenda, 1978)

$$
\boldsymbol{L}_{q P}=\boldsymbol{L}_{P q}
$$

According to the reasons expounded in (Ai, 2006), we may regard the force $\boldsymbol{X}_{P}$ of the region where a ferroelectric phase transition is occurring as a large constant roughly in the characteristic times of phase transition. For the ferroelectric phase transition may be regarded as a stationary state process, the principle of minimum entropy production must be satisfied (Lavenda, 1978)

According to Equations (62), (65), (66), we have

$$
\sigma_{s}=\boldsymbol{L}_{q q}: \boldsymbol{X}_{q} \boldsymbol{X}_{q}+2 \boldsymbol{L}_{q P}: \boldsymbol{X}_{q} \boldsymbol{X}_{P}+\boldsymbol{L}_{P P}: \boldsymbol{X}_{P} \boldsymbol{X}_{P}
$$


If there is no restriction on $\boldsymbol{X}_{q}$ and $\boldsymbol{X}_{P}$, according to the conditions on which the entropy production is a minimum

$$
\begin{aligned}
& \left(\frac{\partial \sigma_{s}}{\partial \boldsymbol{X}_{q}}\right)_{\boldsymbol{X}_{P}}=2 \boldsymbol{L}_{q q} \cdot \boldsymbol{X}_{q}+2 \boldsymbol{L}_{q P} \cdot \boldsymbol{X}_{P}=2 \boldsymbol{J}_{q}=0 \\
& \left(\frac{\partial \sigma_{s}}{\partial \boldsymbol{X}_{P}}\right)_{\boldsymbol{X}_{q}}=2 \boldsymbol{L}_{P q} \cdot \boldsymbol{X}_{q}+2 \boldsymbol{L}_{P P} \cdot \boldsymbol{X}_{P}=2 \boldsymbol{J}_{P}=0
\end{aligned}
$$

we know the stationary states are equilibrium ones actually. If we let $\boldsymbol{X}_{q}$ (or $\boldsymbol{X}_{P}$ ) be a constant, according to Equation (70) (or (69)) we know $\boldsymbol{J}_{P}$ (or $\boldsymbol{J}_{q}$ ), which is corresponded with another force $\boldsymbol{X}_{P}$ (or $\boldsymbol{X}_{q}$ ), should be zero.

Then, a first-order ferroelectric phase transition can be described by the second paradigm. Since the force $\boldsymbol{X}_{P}$ of the region where the phase transition is occurring is a large constant, the flux $\boldsymbol{J}_{q}$ of the region should be zero (but $\boldsymbol{J}_{P} \neq 0$ ). This states clearly that the pure heat conduction and the heat conduction induced by the thermo-electric coupling cancel out each other so as to release or absorb the latent heat. It is certain that the latent heat passes through the region where the phase transition has occurred (at the outside of the region where the phase transition is occurring) and exchanges itself with the thermal bath. Accompanied with the change of the surface's temperature and the unceasing jerky moving of the region where the phase transition is occurring, a constant temperature gradient is kept in the region where the phase transition has occurred, i.e. the force $\boldsymbol{X}_{q}$ is a constant. So, the flux $\boldsymbol{J}_{P}=0$ (but $\left.\boldsymbol{J}_{q} \neq 0\right)$. This states clearly that the electric displacement of the region where the phase transition has occurred will not change but keep the value at Curie temperature or zero until the phase transition finishes. Differently, the region where the phase transition will occur should be described by the first paradigm for there is no restriction on the two forces $\boldsymbol{X}_{P}, \boldsymbol{X}_{q}$. The states of this region are equilibrium ones. So the temperature gradient $\nabla T$ should be zero.

\subsection{Verification of interface dynamics}

Considering that $(\nabla T)_{p a r} \approx 0$ for the region where the phase transition will occur (i.e. the paraelectric phase part) can be regarded as an equilibrium system, we modify equation (46) as

$$
v_{n}=\frac{k_{f e r}(\nabla T)_{f e r} \cdot \boldsymbol{n}}{l \rho}
$$

In order to compare it with experiments, we make use of the following values which are about $\mathrm{PbTiO}_{3}$ crystal: $\rho=7.1 \mathrm{~g} / \mathrm{cm}^{3}$ (Chewasatn \& Milne, 1994), $l=900 \mathrm{cal} / \mathrm{mol}$ (Nomura \& Sawada, 1955), $k_{f e r}=8.8 \times 10^{5} \mathrm{erg} / \mathrm{cm} . \mathrm{s} . \mathrm{K}$ (Mante \& Volger, 1967). The value of the velocity of the interface's fast motion, which has been measured by the experiments, is $0.5 \mathrm{~mm} / \mathrm{s}$ (Dec, 1988). According to equation (71), we calculate the corresponding temperature gradient to be $57.35 \mathrm{~K} / \mathrm{cm}$. However, in (Dec, 1989) it is reported that the experimental temperature gradient varies from 1.5 to $3.5 \mathrm{~K} / \mathrm{mm}$ while the experimental velocity of interface's motion varies from 732 to $843 \mu \mathrm{m} / \mathrm{s}$. Considering the model is rather rough, we may conclude that the theory coincides with the experiments. 


\section{Thermo-electro-mechanical coupling}

The comprehensive thermo-electro-mechanical coupling may be found in the ferroelectric phase transition processes. Because there exists not only the change of polarization but also the changes of system's volume and shape when a ferroelectric phase transition occurs in it, the mechanics can not be ignored even if it is mechanically-free, i.e. no outer force is exerted on it. To a first-order ferroelectric phase transition, it occurs at the surface layer of system firstly, then in the inner part. So, the stress may be found in the system.

Since one aspect of the nature of ferroelectric phase transitions is the thermo-electromechanical coupling, we take the mechanics into account on the basis of Section 4, where only the thermo-electric coupling has been considered. This may lead to a complete description in the sense of continuum physics. In this section, the mathematical deducing is reduced for it is complicated. The details may be referred in (Ai, 2007).

\subsection{Deformation mechanics}

For a continuum, the momentum equation in differential form can be written as

$$
\nabla \cdot \boldsymbol{\sigma}+\rho \boldsymbol{f}=\rho \boldsymbol{a}
$$

where $\boldsymbol{\sigma}, \boldsymbol{f}, \boldsymbol{a}, \rho$ is the stress, the volume force exerted on unit mass, the acceleration and the mass density, respectively. Let $k=\frac{1}{2} v^{2}$ be the local kinetic energy density (per unit mass), with $v$ is the velocity. Then

where $t$ is the time. In terms of

$$
\frac{\mathrm{d} k}{\mathrm{~d} t}=\boldsymbol{v} \cdot \boldsymbol{a}
$$

$$
\begin{gathered}
\nabla \cdot(\boldsymbol{v} \cdot \boldsymbol{\sigma})=\boldsymbol{v} \cdot(\nabla \cdot \boldsymbol{\sigma})+(\nabla \boldsymbol{v}): \boldsymbol{\sigma} \\
\boldsymbol{\sigma}:(\nabla \boldsymbol{v})=\boldsymbol{\sigma}: \boldsymbol{d}
\end{gathered}
$$

we can deduce the following balance equation of mechanical energy basing on Equations (72) (73)

$$
\rho \frac{\mathrm{d} k}{\mathrm{~d} t}-\nabla \cdot(\boldsymbol{v} \cdot \boldsymbol{\sigma})=\rho \boldsymbol{v} \cdot \boldsymbol{f}-\boldsymbol{\sigma}: \boldsymbol{d}
$$

which is in differential form and in Lagrangian form. Or in Eulerian form

$$
\frac{\partial(\rho k)}{\partial t}+\nabla \cdot(\rho k \boldsymbol{v}-\boldsymbol{v} \cdot \boldsymbol{\sigma})=\rho \boldsymbol{v} \cdot \boldsymbol{f}-\boldsymbol{\sigma}: \boldsymbol{d}
$$

where $\boldsymbol{d}=\frac{1}{2}(\nabla \boldsymbol{v}+\boldsymbol{v} \nabla)=\frac{1}{2}\left[\nabla \boldsymbol{v}+(\nabla v)^{T}\right]$ is the rate of deformation or strain rate (the superscript " $\mathrm{T}$ " means transposition).

To a ferroelectric phase transition, $\boldsymbol{f}$ and $\boldsymbol{\sigma}$ may be the nominal volume force and stress, which are the embodiments of the actions of thermo-electro-mechanical coupling and are two inner fields. Generally, they are the sums of real and nominal volume force or stress 


$$
\begin{aligned}
& \boldsymbol{f}=\boldsymbol{f}^{\text {real }}+\boldsymbol{f}^{\text {nom }} \\
& \boldsymbol{\sigma}=\boldsymbol{\sigma}^{\text {real }}+\boldsymbol{\sigma}^{\text {nom }}
\end{aligned}
$$

The nominal volume force and stress are not zero until the eigen (free) deformation of system finishes in phase transitions. If they are zero, the eigen (free) deformation finishes.

\subsection{Local entropy production and description of phase transitions}

The Gibbs equation was given as the following

$$
\mathrm{T} \mathrm{d} s+\sum_{i} \mu_{i} \mathrm{~d} n_{i}+\frac{1}{\rho} \boldsymbol{E} \cdot \mathrm{d} \boldsymbol{D}+\boldsymbol{f} \cdot \mathrm{d} \boldsymbol{r}+\frac{1}{\rho} \nabla \cdot(\boldsymbol{v} \cdot \boldsymbol{\sigma}) \mathrm{d} t=\mathrm{d} u+\mathrm{d} k
$$

where $T, \boldsymbol{E}, \boldsymbol{D}$ is the local temperature, the local electric field intensity and the local electric displacement, respectively; $s, u, n_{i}, \mu_{i}$ is the local entropy density (per unit mass), the local internal energy density (per unit mass), the local molar quantity density (per unit mass) and the chemical potential, respectively; $\boldsymbol{r}$ is the displacement vector. If the outer electric field is not applied, the quantity $\boldsymbol{E}$ is the inner electric field $\boldsymbol{E}^{\text {in }}$ only (Ai, 2006).

Make the material derivative of Equation (80) with $t$, then obtain

$$
\rho \frac{\mathrm{d} s}{\mathrm{~d} t}+\frac{\rho}{T} \sum_{i} \mu_{i} \frac{\mathrm{d} n_{i}}{\mathrm{~d} t}+\frac{1}{T} \boldsymbol{E}^{i n} \cdot \frac{\mathrm{d} \boldsymbol{D}}{\mathrm{d} t}+\frac{\rho}{T} \boldsymbol{f} \cdot \frac{\mathrm{d} \boldsymbol{r}}{\mathrm{d} t}+\frac{1}{T} \nabla \cdot(\boldsymbol{v} \cdot \boldsymbol{\sigma})=\frac{\rho}{T} \frac{\mathrm{d} e}{\mathrm{~d} t}
$$

where $e$ is the total energy with $e=u+k$. We know, the system is in the crystalline states before and after a phase transition so that there is no diffusion of any kind of particles in the system. So, $n_{i}$ as the local molar quantity (per unit mass) does not change with $t$, i.e. $\frac{d n_{i}}{d t}=0 . \frac{d \boldsymbol{D}}{d t}$ stands for the polarization current $\boldsymbol{J}_{P}$, while $\frac{d \boldsymbol{r}}{d t}$ stands for the velocity $\boldsymbol{v}$, $\boldsymbol{E}^{\text {in }}=-\nabla \phi^{\text {in }}$ ( $\phi^{\text {in }}$ stands for the inner electric potential).

After the lengthy and troublesome deduction (Ai, 2007), we obtain the local entropy balance equation in Lagrangian form

$$
\rho \frac{\mathrm{d} s}{\mathrm{~d} t}=-\nabla \cdot \boldsymbol{J}_{s}+\sigma_{s}
$$

with the entropy flux $\boldsymbol{J}_{S}$

$$
\boldsymbol{J}_{s}=\frac{\boldsymbol{J}_{q}^{\text {diff }}+\phi^{i n} \boldsymbol{J}_{P}-\boldsymbol{v} \cdot \boldsymbol{\sigma}}{T}
$$

and the rate of local entropy production $\sigma_{s}$

$$
\sigma_{s}=\boldsymbol{J}_{q}^{\text {diff }} \cdot \nabla\left(\frac{1}{T}\right)+\boldsymbol{J}_{P} \cdot \nabla\left(\frac{\phi^{i n}}{T}\right)-\boldsymbol{\sigma}:\left[\boldsymbol{v} \nabla\left(\frac{1}{T}\right)\right]-\rho \boldsymbol{f} \cdot \frac{\boldsymbol{v}}{T}
$$

where $\boldsymbol{J}_{q}^{\text {diff }}$ is the diffusion of heat, i.e. heat conduction. 
Here are the thermodynamic fluxes $\boldsymbol{J}_{q}^{\text {diff }}, \boldsymbol{J}_{P}, \boldsymbol{\sigma}\left(=\boldsymbol{J}_{\sigma}\right), \rho \boldsymbol{f}\left(=\boldsymbol{J}_{f}\right)$ and the corresponding thermodynamic forces $\boldsymbol{X}_{q}, \boldsymbol{X}_{P}, \boldsymbol{X}_{\sigma}, \boldsymbol{X}_{f}$

$$
\begin{gathered}
\boldsymbol{X}_{q}=\nabla\left(\frac{1}{T}\right) \\
\boldsymbol{X}_{P}=\nabla\left(\frac{\phi^{i n}}{T}\right) \\
\boldsymbol{X}_{\sigma}=-\boldsymbol{v} \nabla\left(\frac{1}{T}\right) \\
\boldsymbol{X}_{f}=-\frac{\boldsymbol{v}}{T}
\end{gathered}
$$

$J_{i}(i=q, P, \sigma, f)$ can be expanded linearly with $X_{j}(j=q, P, \sigma, f)$

$$
\begin{aligned}
& \boldsymbol{J}_{q}=\boldsymbol{L}_{q q} \cdot \boldsymbol{X}_{q}+\boldsymbol{L}_{q P} \cdot \boldsymbol{X}_{P}+\boldsymbol{L}_{q \sigma}: \boldsymbol{X}_{\sigma}+\boldsymbol{L}_{q f} \cdot \boldsymbol{X}_{f} \\
& \boldsymbol{J}_{P}=\boldsymbol{L}_{P q} \cdot \boldsymbol{X}_{q}+\boldsymbol{L}_{P P} \cdot \boldsymbol{X}_{P}+\boldsymbol{L}_{P \sigma}: \boldsymbol{X}_{\sigma}+\boldsymbol{L}_{P f} \cdot \boldsymbol{X}_{f} \\
& \boldsymbol{J}_{\sigma}=\boldsymbol{L}_{\sigma q} \cdot \boldsymbol{X}_{q}+\boldsymbol{L}_{\sigma P} \cdot \boldsymbol{X}_{P}+\boldsymbol{L}_{\sigma \sigma}: \boldsymbol{X}_{\sigma}+\boldsymbol{L}_{\sigma f} \cdot \boldsymbol{X}_{f} \\
& \boldsymbol{J}_{f}=\boldsymbol{L}_{f q} \cdot \boldsymbol{X}_{q}+\boldsymbol{L}_{f P} \cdot \boldsymbol{X}_{P}+\boldsymbol{L}_{f \sigma}: \boldsymbol{X}_{\sigma}+\boldsymbol{L}_{f f} \cdot \boldsymbol{X}_{f}
\end{aligned}
$$

where $\boldsymbol{L}_{q q}, \boldsymbol{L}_{P P}, \boldsymbol{L}_{f f}, \boldsymbol{L}_{q P}, \boldsymbol{L}_{P q}, \boldsymbol{L}_{q f}, \boldsymbol{L}_{f q}, \boldsymbol{L}_{P f}, \boldsymbol{L}_{f P}$ are nine second-order tensors, $\boldsymbol{L}_{q \sigma}, \boldsymbol{L}_{\sigma q}, \boldsymbol{L}_{P \sigma}, \boldsymbol{L}_{\sigma P}, \boldsymbol{L}_{\sigma f}, \boldsymbol{L}_{f \sigma}$ are six third-order tensors, $\boldsymbol{L}_{\sigma \sigma}$ is a fourth-order tensor. The Onsager relations read

$$
L_{i j}=L_{j i}
$$

So the rate of local entropy production can be re-written as

$$
\sigma_{s}=\sum_{i, j=1}^{4} L_{i j} X_{i} X_{j}
$$

For convenience, we have modified the superscripts $q, P, \sigma, f$ to be $1,2,3,4$.

In view of Section 3, the states of a ferroelectric phase transition are stationary. So the principle of minimum entropy production is satisfied. If we keep the former $k$ forces being constants, i.e. $X_{i}=$ const $(i=1,2, \cdots, k, k<4)$, the conditions on which the local entropy production is a minimum are

$$
\left(\frac{\partial \sigma_{s}}{\partial X_{i}}\right)_{X_{j}\left(\neq X_{i}\right)}=\sum_{j=1}^{4}\left(L_{i j}+L_{j i}\right) X_{j}=2 J_{i}=0,(i=k+1, \cdots, 4)
$$


This reveals that the fluxes corresponding to the left $4-k$ forces are zero. Of course, if there are no restrictions on $X_{i}(i=1,2,3,4)$, all the flues are zero.

We may describe a ferroelectric phase transition by using the two paradigms above similarly as do in Section 4. To a first-order ferroelectric phase transition, the forces $\boldsymbol{X}_{P}, \boldsymbol{X}_{\sigma}, \boldsymbol{X}_{f}$ of the region where the phase transition is occurring can be regarded as three large constants roughly in the characteristic times of phase transition because the electric displacement, the volume and the shape change suddenly. So, the flux $\boldsymbol{J}_{q}^{\text {diff }}$ of the region should be zero (but $\boldsymbol{J}_{P} \neq 0, \boldsymbol{\sigma} \neq 0, \rho \boldsymbol{f} \neq 0$ ). This states clearly that the pure heat conduction and the heat conduction induced by the thermo-electric coupling and the thermomechanical coupling cancel out each other so as to release or absorb the latent heat. The phase transition occurs at the surface layer firstly, which is mechanically-free. So, when the phase transition occurs in this region, the flux $\sigma$ maybe the nominal stress $\sigma^{\text {nom }}$ only, which does work to realize the transformation from the internal energy to the kinetic energy. When the phase transition occurs in the inner part, the flux $\sigma$ should be the sum of $\boldsymbol{\sigma}^{\text {real }}$ and $\boldsymbol{\sigma}^{\text {nom }}$ because the sudden changes of inner part's volume and shape have to overcome the bound of outer part then $\sigma^{\text {real }}$ arises. The region where the phase transition is occurring, i.e. the phase boundary is accompanied with the real stress $\sigma^{\text {real }}$ usually, which does work to realize the transformation from the kinetic energy to the internal energy. This has been predicted and described with a propagating stress wave. (Gordon, 1991).

It is certain that the latent heat passes through the region where the phase transition has occurred (at the outside of the region where the phase transition is occurring) and exchange itself with the thermal bath. For $\pm l \rho \boldsymbol{v}_{a}=\boldsymbol{J}_{q}^{\text {diff }}=-\kappa \nabla T$, a constant temperature gradient $\nabla T$ is kept in the region where the phase transition has occurrd, i.e. the force $\boldsymbol{X}_{q}$ at every site is a constant (which does not change with the time but may vary with the position). So, the fluxes $\boldsymbol{J}_{P}=\boldsymbol{\sigma}=\rho \boldsymbol{f}=0$ (but the flux $\boldsymbol{J}_{q}^{\text {diff }} \neq 0$ ). This states clearly that the electric displacement $\boldsymbol{D}$ will not change but keep the value at Curie temperature or zero until the phase transition finishes and $\boldsymbol{\sigma}^{\text {real }}=-\boldsymbol{\sigma}^{\text {nom }}$ in this region. Because the electric displacement $\boldsymbol{D}$ and the strain (or deformation) are all determined by the crystal structure of system, $\boldsymbol{J}_{P}=0$ reveals that $\boldsymbol{D}$ of this region does not change so does not the crystal structure then does not the strain (or deformation). According to (Gordon, 1991), we know the region where the phase transition has occurred is unstressed, i.e. $\sigma^{\text {real }}=0$, then $\sigma^{\text {nom }}=0$. This reveals that the eigen (free) strain (or deformation) of system induced by the thermo-electromechanical coupling of phase transition is complete and the change of it terminates before the phase transition finishes. The two deductions coincide with each other. $\sigma^{\text {real }}$ may relaxes via the free surface.

The region where the phase transition will occur should be in equilibrium because there are no restrictions on the forces $\boldsymbol{X}_{q}, \boldsymbol{X}_{P}, \boldsymbol{X}_{\sigma}, \boldsymbol{X}_{f}$. Whereas, according to (Gordon, 1991), the region is stressed, i.e. $\boldsymbol{\sigma}^{\text {real }} \neq 0$. To the heating process of phase transition, this may lead to a change of the spontaneous polarization of this region because of the electro-mechanical coupling (piezoelectric effect).

An immediate result of the above irreversible thermodynamic description is that the action at a distance, which is the kind of heat transfer at phase transitions, is removed absolutely. The latent heat is transferred within a finite time so the occurrence of phase transition in the inner part is delayed. (Of course, another cause is the stress, just see Section 6) In other 
words, the various parts absorb or release the latent heat at the various times. The action at a distance does not affect the phase transition necessarily.

\subsection{Relation between latent heat and spontaneous polarization}

By now, among the latent heat, the spontaneous polarization, the stress and the strain, only the latent heat and the spontaneous polarization have been measured often for first-order ferroelectric phase transitions. So, we will consider a simplified case of thermo-electric coupling only so as to establish the relation between the latent heat and the spontaneous polarization in the realm of non-equilibrium thermodynamics.

All the quantities of the region where the phase transition has occurred are marked with the superscript "I"; all the quantities of the region where the phase transition is occurring are marked with the superscript "II"; and all the quantities of the region where the phase transition will occur are marked with the superscript "III". Let's consider the heating processes of phase transition firstly. In the region where the phase transition has occurred,

$$
\boldsymbol{J}_{q}^{I-\text { para }}=\boldsymbol{L}_{q q}^{I-\text { para }} \cdot \boldsymbol{X}_{q}^{I-\text { para }}=l \rho \boldsymbol{v}_{a}
$$

where we have ignored the difference between the mass density of ferroelectric phase and that of paraelectric phase (almost the same) and denote them as $\rho, \boldsymbol{v}_{a}$ is the average velocity of interface. In the region where the phase transition is occurring,

$$
\begin{gathered}
\boldsymbol{J}_{q}^{I I}=\boldsymbol{L}_{q q}^{I I} \cdot \boldsymbol{X}_{q}^{I I}+\boldsymbol{L}_{q P}^{I I} \cdot \boldsymbol{X}_{P}^{I I}=0 \\
\boldsymbol{J}_{P}^{I I}=\boldsymbol{L}_{P q}^{I I} \cdot \boldsymbol{X}_{q}^{I I}+\boldsymbol{L}_{P P}^{I I} \cdot \boldsymbol{X}_{P}^{I I}
\end{gathered}
$$

The heat which is transferred to the region where the phase transition is occurring is absorbed as latent heat because the pure heat conduction and the heat conduction induced by the thermo-electric coupling cancel out each other. So,

$$
l \rho \boldsymbol{v}_{a}=\boldsymbol{L}_{q q}^{I I} \cdot \boldsymbol{X}_{q}^{I I}
$$

According to Eq. (97)-(99), we work out

$$
\boldsymbol{J}_{P}^{I I}=l \rho\left[\boldsymbol{L}_{P q}^{I I} \cdot\left(\boldsymbol{L}_{q q}^{I I}\right)^{-1}-\boldsymbol{L}_{P P}^{I I} \cdot\left(\boldsymbol{L}_{q P}^{I I}\right)^{-1}\right] \cdot \boldsymbol{v}_{a}
$$

where the superscript " -1 " means reverse. While

$$
\int \boldsymbol{J}_{P}^{I I} \mathrm{~d} t=-\boldsymbol{D}^{\text {spon }}=\varepsilon_{0} \nabla \phi^{i n-I I I}-\boldsymbol{P}^{\text {spon-III }}=-\boldsymbol{P}^{\text {spon-III }}
$$

where we utilized the boundary condition of $\boldsymbol{D}$ and considered the region where the phase transition will occur is in equilibrium, the superscript "spon" means spontaneous. So,

$$
\boldsymbol{P}^{\text {spon }}=\boldsymbol{P}^{\text {spon-III }}=-\int \boldsymbol{J}_{P}^{I I} \mathrm{~d} t
$$

The relation between the latent heat and the spontaneous polarization are obtained. In the cooling processes of phase transition, 


$$
\begin{gathered}
-l \rho \boldsymbol{v}_{a}=\boldsymbol{L}_{q q}^{I-f e r r} \cdot \boldsymbol{X}_{q}^{I-\text { ferr }}+\boldsymbol{L}_{q P}^{I-\text { ferr }} \cdot \boldsymbol{X}_{P}^{I-\text { ferr }}=\boldsymbol{L}_{q q}^{I I} \cdot \boldsymbol{X}_{q}^{I I} \\
\int \boldsymbol{J}_{P}^{I I^{\prime}} \mathrm{d} t=\boldsymbol{D}^{\text {spon-I }}=-\varepsilon_{0} \nabla \phi^{i n-I}+\boldsymbol{P}^{\text {spon-I }}
\end{gathered}
$$

Repeating the above steps, we obtain

$$
\boldsymbol{P}^{\text {spon-I }}=\int \boldsymbol{J}_{P}^{I I^{\prime}} \mathrm{d} t+\varepsilon_{0} \nabla \phi^{i n-I}
$$

with

$$
\boldsymbol{J}_{P}^{I I^{\prime}}=-l \rho\left[\boldsymbol{L}_{P q}^{I I} \cdot\left(\boldsymbol{L}_{q q}^{I I}\right)^{-1}-\boldsymbol{L}_{P P}^{I I} \cdot\left(\boldsymbol{L}_{q P}^{I I}\right)^{-1}\right] \cdot \boldsymbol{v}_{a}=-\boldsymbol{J}_{P}^{I I}
$$

Then we find that $\boldsymbol{P}^{\text {spon-III }}$ (serves as the equilibrium polarization) is not equal to $\boldsymbol{P}^{\text {spon-I }}$ (serves as the non-equilibrium polarization). For the region where the phase transition will occur is stressed, there is some difference between $\boldsymbol{P}^{\text {spon }}\left(=\boldsymbol{P}^{\text {spon-III }}\right)$ and the equilibrium spontaneous polarization without the affects of stress $\boldsymbol{P}^{\text {spon }}$ because of the piezoelectric effect.

\section{Irreversibility: thermal hysteresis and occurrences of domain structure}

\subsection{Thermal hysteresis}

The "thermal hysteresis" of first-order ferroelectric phase transitions is an irreversible phenomenon obviously. But it was treated by using the equilibrium thermodynamics for ferroelectric phase transitions, the well-known Landau-Devonshire theory (Lines \& Glass,1977). So, there is an inherent contradiction in this case. The system in which a firstorder ferroelectric phase transition occurs is heterogeneous. The occurrences of phase transition in different parts are not at the same time. The phase transition occurs at the surface layer then in the inner part of system. According to the description above, we know a constant temperature gradient is kept in the region where the phase transition has occurred. The temperature of surface layer, which is usually regarded as the temperature of the whole system in experiments, must be higher (or lower) than the Curie temperature. This may lead to the thermal hysteresis.

No doubt that the shape and the area of surface can greatly affect the above processes. We may conclude that the thermal hysteresis can be reduced if the system has a larger specific surface and, the thermal hysteresis can be neglected if a finite system has an extremely-large specific surface. So, the thermal hysteresis is not an intrinsic property of the system.

The region where the phase transition will occur can be regarded as an equilibrium system for there are no restrictions on the forces $\boldsymbol{X}_{q}, \boldsymbol{X}_{P}, \boldsymbol{X}_{\sigma}, \boldsymbol{X}_{f}$. In other words, the forces and the corresponding fluxes are zero in this region. To a system where a second-order ferroelectric phase transition occurs, the case is somewhat like that of the region where a first-order ferroelectric phase transition will occur. The spontaneous polarization, the volume and the shape of system are continuous at the Curie temperature and change with the infinitesimal magnitudes. This means $\boldsymbol{X}_{q}, \boldsymbol{X}_{P}, \boldsymbol{X}_{\sigma}, \boldsymbol{X}_{f}$ and $\boldsymbol{J}_{q}^{\text {diff }}, \boldsymbol{J}_{P}, \boldsymbol{\sigma}, \rho \boldsymbol{f}$ can be arbitrary infinitesimal magnitudes. The second-order phase transition occurs in every part of the system 
simultaneously, i.e. there is no the co-existence of two phases (ferroelectric and paraelectric). So, there is no the latent heat and stress. The thermal hysteresis disappears.

The region where a first-order ferroelectric phase transition will occur is stressed. This reveals that the occurrences of phase transition in the inner part have to overcome the bound of outer part, where the phase transition occurs earlier. This may lead to the delay of phase transition in the inner part.

\subsection{Occurrences of domain structure}

Though the rationalization of the existence of domain structures can be explained by the equilibrium thermodynamics, the evolving characteristics of domain occurrences in ferroelectrics can not be explained by it, but can be explained by the non-equilibrium thermodynamics.

In the region where the phase transition is occurring, the thermodynamic forces $\boldsymbol{X}_{\boldsymbol{P}}\left(=\nabla\left(\frac{\phi^{i n}}{T}\right)\right), \boldsymbol{X}_{\sigma}\left(=-\boldsymbol{v} \nabla\left(\frac{1}{T}\right)\right), \boldsymbol{X}_{\boldsymbol{f}}\left(=-\frac{\boldsymbol{v}}{T}\right)$ can be regarded as three large constants in the characteristic time of transition and the thermodynamic flux $\boldsymbol{J}_{\boldsymbol{q}}^{\text {diff }}=0$ (but $\boldsymbol{J}_{\boldsymbol{P}} \neq 0, \sigma \neq 0$, $\rho \boldsymbol{f} \neq 0$ ). The local entropy production (cf. Equation (84)) reduces to

$$
\sigma_{s}=\boldsymbol{J}_{\boldsymbol{P}} \cdot \nabla\left(\frac{\phi^{i n}}{T}\right)-\sigma:\left[\boldsymbol{v} \nabla\left(\frac{1}{T}\right)\right]-\rho \boldsymbol{f} \cdot \frac{\boldsymbol{v}}{T}
$$

Now, we are facing a set of complicated fields of $T, \boldsymbol{v}$ and $\phi^{\text {in }}$, respectively. Assume that the phase transition front is denoted by $S$. The points included in $S$ stand for the locations where the transition is occurring. Because the transition occurs along all directions from the outer part to the inner part, we may infer that the orientations of $\nabla\left(\frac{\phi^{i n}}{T}\right)$, or of $-v \nabla\left(\frac{1}{T}\right)$ and or of $-\frac{\boldsymbol{v}}{T}$ vary continuously such that they are differently oriented at defferent locations. There are always several (at least two) symmetry equivalent orientations in the prototype phase (in most cases it is the high temperature phase), which are the possible orientations for spontaneous polarization (or spontaneous deformation or spontaneous displacement). Therefore, the spontaneous polarization, the spontaneous deformation and the spontaneous displacement must take an appropriate orientation respectively to ensure $\sigma_{s}$ is a positive minimum when the system transforms from the prototype (paraelectric) phase to the ferroelectric (low temperature) phase. The underlying reasons are that

$$
\begin{gathered}
\boldsymbol{J}_{P}=\frac{\mathrm{d} \boldsymbol{D}}{\mathrm{d} t}=\frac{\mathrm{d}\left(\varepsilon_{0} \boldsymbol{E}^{i n}+\boldsymbol{P}\right)}{\mathrm{d} t} \\
\sigma=\boldsymbol{L}: \boldsymbol{\varepsilon} \\
\rho \boldsymbol{f}=\rho \frac{\mathrm{d} \boldsymbol{v}}{\mathrm{d} t}-\nabla \cdot \sigma
\end{gathered}
$$


where $\boldsymbol{L}, \varepsilon$, and $\varepsilon_{0}$ is the modulus of rigidity, the strain and the permittivity of vacuum, respectively. Therefore, $\boldsymbol{P}, \varepsilon$ at different locations will be differently oriented. The domain structures in ferroelectrics thus occur.

It seems that the picture of domain occurrences for first-order ferroelectric phase transition systems should disappear when we face second-order ferroelectric phase transition systems. This is true if the transition processes proceed infinitely slowly as expounded by the equilibrium thermodynamics. But any actual process proceeds with finite rate, so it is irreversible. Then the above picture revives.

In (Ai et al., 2010), the domain occurrences in ferromagnetics can be described parallelly by analogy. And the case of ferroelastic domain occurrences is a reduced, simpler one compared with that of ferroelectrics or ferromagnetics.

It is well known that the Landau theory or the Curie principle tells us how to determine the symmetry change at a phase transition. A concise statement is as follows (Janovec, 1976): for a crystal undergoing a phase transition with a space-group symmetry reduction from $G_{0}$ to $G$, whereas $G$ determines the symmetry of transition parameter (or vice versa), it is the symmetry operations lost in going from $G_{0}$ to $G$ that determine the domain structure in the lowsymmetry phase. The ferroic phase transitions are the ones accompanied by a change of point group symmetry (Wadhawan, 1982). Therefore, the substitution of "point group" for the "space group" in the above statement will be adequate for ferrioc phase transitions. From the above statement, the domain structure is a manifestation of the symmetry operations lost at the phase transition. In our treatment of the domain occurrences in ferroics, we took into account the finiteness of system (i.e. existence of surface) and the irreversibility of process (asymmetry of time). The finiteness of system makes the thermodynamic forces such as $\nabla\left(\frac{\phi^{i n}}{T}\right),-v \nabla\left(\frac{1}{T}\right),-\frac{v}{T}$ have infinite space symmetry. The infinite space symmetry, combined with the asymmetry of time, reproduces the symmetry operations lost at the phase transition in the ferroic phase. It can be viewed as an embodiment of time-space symmetry.

After all, for the domain structures can exist in equilibrium systems, they are the equilibrium structures but not the dissipative ones, for the latter can only exist in systems far from equilibrium (Glansdorff \& Prigogine, 1971).

\section{Acknowledgments}

I acknowledge the supports of the Natural Science Foundation Programs of Shandong Province (Grant No. Y2008A36).

\section{References}

Ai, S.T. (2006). Paraelectric-Ferroelectric Interface Dynamics Induced by Latent Heat Transfer and Irreversible Thermodynamics of Ferroelectric Phase Transitions. Ferroelectrics, Vol. 345, No.1, (June, 2006) 59-66, ISSN 1563-5112 (online), 0015-0193 (print).

Ai, S.T. (2007). Mechanical-Thermal-Electric Coupling and Irreversibility of Ferroelectric Phase Transitions. Ferroelectrics, Vol.350, No.1 (May, 2007) 81-92, ISSN 1563-5112 (online), 0015-0193 (print).

Ai, S.T.; Xu, C.T.; Wang, Y.L.; Zhang, S.Y.; Ning, X.F. \& Noll, E. (2008). Comparison of and Comments on Two Thermodynamic Approaches (Reversible and Irreversible) to 
Ferroelectric Phase Transitions. Phase Transit., Vol.81, No.5, (May, 2008) 479-490, ISSN 1029-0338(online), 0141-1594 (print).

Ai, S.T.; Zhang, S.Y.; Ning, X.F.; Wang, Y.L. \& Xu C.T. (2010). Non-Equilibrium Thermodynamic Explanation of Domain Occurrences in Ferroics. Ferroelectrics Lett., Vol.37, No.2, (March, 2010) 30-34, ISSN 1563-5228 (online), 0731-5171 (print).

Chewasatn, S. \& Milne, S.T. (1994). Synthesis and Characterization of $\mathrm{PbTiO}_{3}$ and $\mathrm{Ca}$ and Mn Modified $\mathrm{PbTiO}_{3}$ Fibres Produced by Extrusion of Diol Based Gels. J. Mat. Sci., Vol.29, No.14, (January, 1994) 3621-3629, ISSN 1573-4803 (online), 0022-2461 (print).

Dec, J. (1988). Jerky Phase-Front Motion in $\mathrm{PbTiO}_{3}$ Crystal. J. Phys. C, Vol.21, No.7, (March, 1988) 1257-1263, ISSN 0022-3719 (print).

Dec, J. (1989). The Phase Boundary as a Kink. Ferroelectrics, Vol.89, No.1, (January, 1989) 193200, ISSN 1563-5112 (online), 0015-0193 (print).

Dec, J. \& Yurkevich, J. (1990). The Antiferroelectric Phase Boundary as a Kink. Ferroelectrics, Vol. 110, No.1, (October, 1990) 77-83, ISSN 1563-5112 (online), 0015-0193 (print).

Devonshire, A.F. (1949). Theory of Barium Titanate (Part I). Phil. Mag., Vol.40, No.10, (October, 1949) 1040-1063, ISSN 1478-6443 (online), 1478-6435(print).

Devonshire, A.F. (1951). Theory of Barium Titanate (Part II). Phil. Mag., Vol.42, No.10, (October, 1951) 1065-1079, ISSN 1478-6443 (online), 1478-6435(print).

Devonshire, A.F. (1954). Theory of Ferroelectrics. Adv. Phys., Vol.3, No.2, (May, 1954) 85-130, ISSN 1460-6976 (online), 0001-8732 (print).

Glansdorff, P. \& Prigogine, I. (1971). Thermodynamic Theory of Structure, Stability and Fluctuations, Wiley-Interscience, ISBN 978-0471302803, New York.

Gordon, A. (1991). Propagation of Solitary Stress Wave at First-Order Ferroelectric Phase Transitions. Phys. Lett. A, Vol.154, No.1-2, (March, 1991) 79-80, ISSN 0375-9601.

Gordon, A. (2001). Finite-Size Effects in Dynamics of Paraelectric-Ferroelectric Interfaces Induced by Latent Heat Transfer. Phys. Lett. A, Vol.281, No.5-6, (April, 2001) 357362, ISSN 0375-9601.

Gordon, A.; Dorfman, S. \& Fuks, D. (2002). Temperature-Induced Motion of Interface Boundaries in Confined Ferroelectrics. Phil. Mag. B, Vol.82, No.1, (January, 2002) 63-71, ISSN 1463-6417 (online), 1364-2812 (print).

Janovec, V. (1976). A Symmetry Approach to Domain Structures. Ferroelectrics, Vol.12, No.1, (June, 1976) 43-53, ISSN 1563-5112 (online), 0015-0193 (print).

Lavenda, B.H. (1978). Thermodynamics of Irreversible Processes, Macmillan, ISBN 0-333-216164, London and Basingstoke.

Lines, M.E. \& Glass, A.M. (1977). Principles and Applications of Ferroelectrics and Related Materials, Clarendon, ISBN 978-0198507789, Oxford.

Mante, A.J.H. \& Volger, J. (1967). The Thermal Conductivity of $\mathrm{PbTiO}_{3}$ in the Neighbourhood of its Ferroelectric Transition Temperature. Phys. Lett. A, Vol.24, No.3, (January, 1967) 139-140, ISSN 0375-9601.

Nomura, S. \& Sawada, S. (1955). Dielectric Properties of Lead-Strontium Titanate. J. Phys. Soc. Japan, Vol.10, No.2, (February, 1955) 108-111, ISSN 1347-4073 (online), 00319015 (print).

Wadhawan, V.K. (1982). Ferroelasticity and Related Properties of Crystals. Phase Transit., Vol.3, No.1, (March, 1982) 3-103, ISSN 1029-0338 (online), 0141-1594 (print).

Yufatova, S.M.; Sindeyev, Y.G.; Garilyatchenko, V.G. \& Fesenko, E.G. (1980). Different Kinetics Types of Phase Transformations in Lead Titanate. Ferroelectrics, Vol.26, No.1 (January, 1980) 809-812, ISSN 1563-5112 (online), 0015-0193 (print).

Zhong, W.L. (1996). Physics of Ferroelectrics (in Chinese), Science Press, ISBN 7-03-005033-9/O. 834, Beijing. 


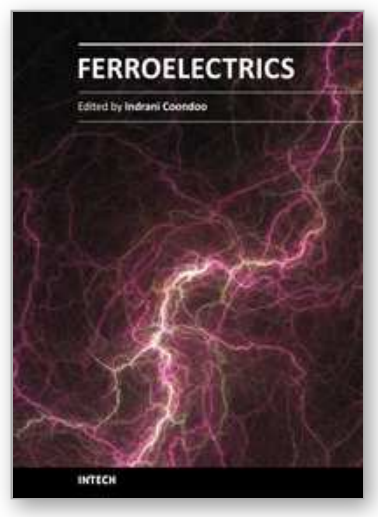

\author{
Ferroelectrics \\ Edited by Dr Indrani Coondoo
}

ISBN 978-953-307-439-9

Hard cover, 450 pages

Publisher InTech

Published online 14, December, 2010

Published in print edition December, 2010

Ferroelectric materials exhibit a wide spectrum of functional properties, including switchable polarization, piezoelectricity, high non-linear optical activity, pyroelectricity, and non-linear dielectric behaviour. These properties are crucial for application in electronic devices such as sensors, microactuators, infrared detectors, microwave phase filters and, non-volatile memories. This unique combination of properties of ferroelectric materials has attracted researchers and engineers for a long time. This book reviews a wide range of diverse topics related to the phenomenon of ferroelectricity (in the bulk as well as thin film form) and provides a forum for scientists, engineers, and students working in this field. The present book containing 24 chapters is a result of contributions of experts from international scientific community working in different aspects of ferroelectricity related to experimental and theoretical work aimed at the understanding of ferroelectricity and their utilization in devices. It provides an up-to-date insightful coverage to the recent advances in the synthesis, characterization, functional properties and potential device applications in specialized areas.

\title{
How to reference
}

In order to correctly reference this scholarly work, feel free to copy and paste the following:

Shu-tao Ai (2010). Non-Equilibrium Thermodynamics of Ferroelectric Phase Transitions, Ferroelectrics, Dr Indrani Coondoo (Ed.), ISBN: 978-953-307-439-9, InTech, Available from:

http://www.intechopen.com/books/ferroelectrics/non-equilibrium-thermodynamics-of-ferroelectric-phasetransitions

\section{INTECH}

open science | open minds

\section{InTech Europe}

University Campus STeP Ri

Slavka Krautzeka 83/A

51000 Rijeka, Croatia

Phone: +385 (51) 770447

Fax: +385 (51) 686166

www.intechopen.com

\section{InTech China}

Unit 405, Office Block, Hotel Equatorial Shanghai

No.65, Yan An Road (West), Shanghai, 200040, China

中国上海市延安西路65号上海国际贵都大饭店办公楼 405 单元

Phone: +86-21-62489820

Fax: +86-21-62489821 
(C) 2010 The Author(s). Licensee IntechOpen. This chapter is distributed under the terms of the Creative Commons Attribution-NonCommercialShareAlike-3.0 License, which permits use, distribution and reproduction for non-commercial purposes, provided the original is properly cited and derivative works building on this content are distributed under the same license. 